TRANSACTIONS OF THE

AMERICAN MATHEMATICAL SOCIETY

Volume 362, Number 4, April 2010, Pages 1963-1979

S 0002-9947(09)04971-X

Article electronically published on November 13, 2009

\title{
EMBEDDING THEOREMS AND BOUNDARY-VALUE PROBLEMS FOR CUSP DOMAINS
}

\author{
V. GOL'DSHTEIN AND M. JU. VASILTCHIK
}

\begin{abstract}
We study the Robin boundary-value problem for bounded domains with isolated singularities. Because trace spaces of space $W_{2}^{1}(D)$ on boundaries of such domains are weighted Sobolev spaces $L^{2, \xi}(\partial D)$, existence and uniqueness of corresponding Robin boundary-value problems depends on properties of embedding operators $I_{1}: W_{2}^{1}(D) \rightarrow L^{2}(D)$ and $I_{2}: W_{2}^{1}(D) \rightarrow$ $L^{2, \xi}(\partial D)$ i.e. on types of singularities. We obtain an exact description of weights $\xi$ for bounded domains with 'outside peaks' on its boundaries. This result allows us to formulate correctly the corresponding Robin boundaryvalue problems for elliptic operators. Using compactness of embedding operators $I_{1}, I_{2}$, we prove also that these Robin boundary-value problems with the spectral parameter are of Fredholm type.
\end{abstract}

\section{INTRODUCTION}

An essentially self-contained presentation of a method for a correct formulation and an investigation of the Robin boundary-value problem for second order elliptic equations in domains with 'outside peaks' is given in this paper. This study is initiated by the works [12, [6, 8]. Quoted papers are devoted to the Robin boundary-value problem for Lipschitz domains and its straightforward generalizations. It is well known that the embedding operator $I_{1}: W_{2}^{1}(D) \rightarrow L^{2}(D)$ is compact for Lipschitz domains 11 and by 8 the operator $I_{2}: W_{2}^{1}(D) \rightarrow L^{2}(\partial D)$ is also compact. Therefore the Robin boundary-value problem is of Fredholm type for this class of domains [8].

For domains with isolated singularities the second embedding operator $I_{2}$ : $W_{2}^{1}(D) \rightarrow L^{2}(\partial D)$ does not necessarily exist, because traces of functions from $W_{2}^{1}(D)$ do not necessarily belong to the space $L^{2}(\partial D)$. This means that even a correct formulation of the Robin problem for such domains depends on properties of a trace space of $W_{2}^{1}(D)$. One of the possible descriptions of trace spaces for bounded $C^{1}$-domains with isolated singularities of an 'outside peak' type was proposed in [14, 15] by the second author. For such domains the trace space for $W_{2}^{1}(D)$ does not necessarily coincide with $L^{2}(D)$ and can be described with the help of corresponding weights $\xi$ that depend on singularity types. These results allow us to formulate correctly the Robin boundary-value problem with the help of the weights $\xi$.

Received by the editors October 9, 2007.

2000 Mathematics Subject Classification. Primary 46E35, 35J25.

The first author was supported in part by the Israel Science Foundation grant.

The second author was partially supported by the Russian Foundation for Basic Research (grant 06-01-00735). 
We will prove in this paper that embedding operators $I_{2}: W_{2}^{1}(D) \rightarrow L^{2, \xi}(\partial D)$ are compact for such weights. Because both embedding operators $I_{1}: W_{2}^{1}(D) \rightarrow$ $L^{2}(D)$ and $I_{2}: W_{2}^{1}(D) \rightarrow L^{2, \xi}(\partial D)$ are compact, the Robin boundary-value problem with the spectral parameter is of Fredholm type.

Elliptic boundary-value problems were studied in numerous books and papers. We mention [4 and 10, where many references can be found. In [11] embedding theorems for a variety of domains with nonsmooth boundaries have been studied. The main results of this paper were announced in [16].

Remark. We refer in this paper to domains of class $C^{1}$. We use this condition for simplicity only. All results are also correct for the Lipschitz category.

\section{MAin Results}

In this section we introduce necessary definitions and formulate main results.

Recall the classical definition of Sobolev space $W_{p}^{1}(D)$. Let $D$ be an open subset of $\mathbb{R}^{n}, n \geq 2$. Define Sobolev space $W_{p}^{1}(D), 1 \leq p<\infty$ as a normed space of locally summable, weakly differentiable functions $f: D \rightarrow \mathbb{R}$ equipped with the following norm:

$$
\left\|f \mid W_{p}^{1}(D)\right\|=\left[\left(\int_{D}|f|^{p}(x) d x\right)^{2 / p}+\left(\int_{D}|\nabla f|^{p}(x) d x\right)^{2 / p}\right]^{1 / 2} .
$$

Here $\nabla f$ is the weak gradient of the function $f$.

2.1. Weak Robin problem for bounded $C^{1}$-domains. Let $G \in R^{n}$ be a bounded $C^{1}$-domain i.e. its boundary $\partial G$ is a compact $(n-1)$-dimensional $C^{1}$ manifold. Recall the classical Robin boundary-value problem

$$
\begin{aligned}
& \sum_{i, j=1}^{n} \frac{\partial}{\partial x_{i}}\left(a_{i j}(x) \frac{\partial u}{\partial x_{j}}\right)+\sum_{i=1}^{n} b_{i}(x) \frac{\partial u}{\partial x_{i}}+a(x) u=f(x), x \in G, \\
& \frac{\partial u}{\partial N}+\sigma(x) u=\mu(x), x \in \partial G .
\end{aligned}
$$

Here the functions $a_{i, j}(x)=a_{j, i}, b_{i}(x), a(x), f(x)$ are $C^{1}$-functions defined on $G$ and $\sigma(x), \mu(x)$ are $C^{1}$-functions defined on $\partial G$.

As usual, $\frac{\partial u}{\partial N}=\sum_{i, j=1}^{n} a_{i j} \frac{\partial u}{\partial x_{j}} \cos \left(\vec{n}, x_{i}\right)$, where $\vec{n}$ is the exterior unit normal vector at a point $x \in \partial G$.

Suppose also that the following condition of uniform ellipticity holds:

$$
C_{1}|\xi|^{2} \leq \sum_{i, j=1}^{n} a_{i j}(x) \xi_{i} \xi_{j} \leq C_{2}|\xi|^{2}
$$

for all $\xi=\left(\xi_{1}, \ldots, \xi_{n}\right) \in \mathbb{R}^{n}$. Here $C_{1}$ and $C_{2}$ are positive constants. 
This formulation is equivalent to the following weak formulation. A function $u \in W_{2}^{1}(G)$ is a weak solution of the Robin problem if

$$
\begin{array}{r}
\sum_{i, j=1}^{n} \int_{G} a_{i j}(x) \frac{\partial u}{\partial x_{j}} \frac{\partial \bar{\eta}}{\partial x_{i}} d x-\sum_{i=1}^{n} \int_{G} b_{i}(x) \frac{\partial u}{\partial x_{i}} \bar{\eta} d x-\int_{G} a u \bar{\eta} d x \\
=-\int_{G} f \bar{\eta} d x-\int_{\partial G} \sigma u \bar{\eta} d S_{x}+\int_{\partial G} \mu \bar{\eta} d S_{x}
\end{array}
$$

for all $\eta \in W_{2}^{1}(G)$. Here $d S_{x}$ is the standard surface measure.

For the weak formulation, smoothness of functions $f, \sigma, \mu$ is not necessary and can be replaced by the following weak conditions: $a_{i, j}, b_{i}, a \in L^{\infty}(G), f \in L^{2}(G)$, $\sigma \in L^{\infty}(\partial G), \mu \in L^{2}(\partial G)$.

2.2. Weak Robin problem for domains with an 'outside peak'. Suppose domain $G$ is not of class $C^{1}$ at one isolated point.

Assumptions on the functions $a_{i j}=a_{j i}, f$ and $a$ are the same for any such domain: $f \in L^{2}(G), a \in L^{\infty}$ and $a_{i j}=a_{j i} \in L^{\infty}(G)$. Additional conditions for $b_{i}$, $\sigma, \mu$ depend on properties of the boundary and will be formulated later only for domains with 'outside peaks'.

The next definition is a formal description of domains with an 'outside peak'.

Definition 2.1. We call a bounded $C^{1}$-domain $G \subset \mathbb{R}^{n}$ a domain of class $O P_{\varphi}$ if

1. There exists such point $O \in \partial G$ that $\partial G \backslash\{O\}$ is an $(n-1)$-dimensional $C^{1}$-manifold.

2. Let $\Omega \subset \mathbb{R}^{n-1}$ be a bounded domain of the class $C^{1}$ and function $\varphi \in$ $C^{1}([0,1])$ be such that $\varphi(0)=\varphi^{\prime}(0)=0$ and $\varphi^{\prime}(t)>0$ for $t \in(0,1)$. Denote $x^{\prime}=\left(x_{1}, \ldots, x_{n-1}\right)$.

There exists a neighborhood $U(O)$ of $O$ that can be represented as

$$
U(O) \cap G=\left\{x=\left(x^{\prime}, x_{n}\right) \in \mathbb{R}^{n}: 0<x_{n}<1, \frac{x^{\prime}}{\varphi\left(x_{n}\right)} \in \Omega\right\}
$$

for an appropriate choice of a coordinate system with the origin $O$ in $\mathbb{R}^{n}$.

The point $O$ is a top of an 'outside peak'. We will study problem (2.4) for domains of class $O P_{\varphi}$.

Denote by $L^{p, \xi}(\partial G)$ such a space of measurable functions defined on $\partial G$ that

$$
\int_{\partial G}|f(x)|^{p} \xi(x) d S_{x} \equiv\|f\|_{p, \xi, \partial G}^{p}<\infty .
$$

Here $\xi: \partial G \rightarrow R$ is a fixed nonnegative measurable function (a weight).

We are ready to specify all necessary assumptions for Robin boundary-value problem (2.4) under the uniform ellipticity condition (2.3) for domains of class $O P_{\varphi}$.

Suppose domain $G$ belongs to the class $O P_{\varphi}$. Conditions for functions $a_{i j}, b_{i}, a, f$ are the same as for the weak Robin problem for bounded $C^{1}$-domains. Assumptions for functions $\sigma, \mu$ are different and depend on function $\varphi$.

The functions $\sigma, \mu: \partial G \rightarrow R$ satisfy following conditions:

$$
\operatorname{ess}_{\sup _{x \in \partial G}} \frac{|\sigma(x)|}{\varphi\left(x_{n}\right)}=M_{\sigma}<\infty, \mu \in L^{2, \frac{1}{\varphi}}(\partial G) \text {. }
$$


Here constant $M_{\sigma}$ depends only on function $\varphi$ that describes the singularity type. The condition for $\mu$ is equivalent to $\frac{\mu}{\varphi} \in L^{2, \varphi}(\partial G)$.

New assumptions for $\sigma, \mu$ are essential only in a neighborhood of singularity point $O \in \partial G$. Roughly speaking they correlate with the exact description of the trace space of $W_{2}^{1}(G)$ on the boundary $\partial G$. Reasons for these assumptions will be made clear during the proofs of the main results.

Recall that functions $b_{i}, a, f, u, \eta$ (see (2.4)) are complex-valued functions.

2.3. Main results. Let $I_{1}$ be the embedding operator of $W_{2}^{1}(G)$ into $L^{2}(G)$, and let $I_{2}$ be the embedding operator of $W_{2}^{1}(G)$ into $L^{2, \varphi}(\partial G)$. By 14 the space $L^{2, \varphi}(\partial G)$ contains traces of $W_{2}^{1}(G)$ on $\partial G$. Existence, boundedness and compactness of the operator $I_{1}$ are well known (see, for example, [11). Existence and boundedness of the operator $I_{2}$ are proved in [14. Proving compactness of $I_{2}$ is the main technical goal of this paper.

Remember that $W_{2}^{1}(G)$ with an inner product

$$
[u, \eta]=\int_{G}\left[\sum_{i, j=1}^{n} \frac{\partial u}{\partial x_{i}} \frac{\partial \bar{\eta}}{\partial x_{j}}+u \bar{\eta}\right] d x
$$

is a Hilbert space.

We adopt a general well-known statement of functional analysis to our study.

Proposition 2.2. Suppose $G \in O P_{\varphi}$ and operators $I_{1}$ and $I_{2}$ are compact. Then the weak Robin problem (2.4)-(2.6) is of Fredholm type; i.e., the problem can be reduced to an operator equation on $W_{2}^{1}(G)$,

$$
(I+A) u=F \text {, }
$$

where $I$ is the identity and $A$ is a compact operator.

Compactness of the embedding operator $I_{2}$ is the content of the following result:

Theorem 2.3. If $G \in O P_{\varphi}$, then the embedding operator $I_{2}$ is compact.

This theorem is a special case, $p=2$, of the corresponding result for Sobolev spaces $W_{p}^{1}(G)$ that will be proved in Section 4. Combining two previous results and using compactness of the embedding operator $I_{1}$ [11] we obtain one of the main results of this study.

Theorem 2.4. If $G \in O P_{\varphi}$, then the weak Robin problem (2.4)-(2.6) is of Fredholm type.

Proof of the main results is based on the exact descriptions of the trace spaces of Sobolev spaces $W_{p}^{1}(G)$ on boundaries of $O P_{\varphi}$-domains [14]. For the reader's convenience we reproduce this description here.

Furthermore, the relation $A \sim B$ means that a two-sided inequality $C_{1}<\frac{A}{B}<C_{2}$ with constants $0<C_{1}<C_{2}<\infty$ depending only on $G$ is correct.

Denote by $T W_{p}^{1}(G)$ a normed space of $W_{p}^{1}(G)$-function traces on $\partial G$ with the quotient norm

$$
\|f\|_{T_{p}^{1}(G)}=\inf \left\{\|F\|_{W_{p}^{1}(G)}: F \in W_{p}^{1}(G),\left.F\right|_{\partial G}=f\right\} .
$$

Let $E(x, y)=\max \left\{\varphi\left(x_{n}\right), \varphi\left(y_{n}\right)\right\}$ and $\sigma(x, y)=\chi\left(\frac{|x-y|}{E(x, y)}\right)$, where $\chi$ is the indicator of $[0 ; 1]$. 
Let $G \in O P_{\varphi}, 1<p<\infty$. By [14,

$$
\|f\|_{T W_{p}^{1}} \sim\|f\|_{p, \varphi, \partial G}+\left\{\iint_{G \times G} \frac{|f(x)-f(y)|^{p}}{|x-y|^{n+p-2}} \sigma(x, y) d S_{x} d S_{y}\right\}^{1 / p} .
$$

This means, in particular, that $f / \partial G \in L^{p, \varphi}$.

\section{Proof of Proposition 2.2}

Proof. The space $W_{2}^{1}(G)$ is a Hilbert space with the inner product (2.7). Because of the condition (2.3) this inner product is equivalent to the following new one:

$$
[u, \eta]=\int_{G}\left[\sum_{i, j=1}^{n} a_{i j}(x) \frac{\partial u}{\partial x_{i}} \frac{\partial \bar{\eta}}{\partial x_{j}}+u \bar{\eta}\right] d x
$$

Rewrite the weak Robin boundary problem in terms of this inner product as

$[u, \eta]-\sum_{i=1}^{n} \int_{G} b_{i}(x) \frac{\partial u}{\partial x_{i}} \bar{\eta} d x-\int_{G}(a+1) u \bar{\eta} d x+\int_{\partial G} \sigma u \bar{\eta} d S_{x}=-\int_{G} f \bar{\eta} d x+\int_{\partial G} \mu \bar{\eta} d S_{x}$.

Every integral in (3.2) can be considered as a complex-valued linear functional on $W_{2}^{1}(G)$ :

$$
\begin{aligned}
l_{1, u}(\eta) & =-\sum_{i=1}^{n} \int_{G} b_{i}(x) \frac{\partial u}{\partial x_{i}}(x) \bar{\eta}(x) d x, \quad l_{2, u}(\eta)=-\int_{G}(a+1) u(x) \bar{\eta}(x) d x, \\
l_{3, u}(\eta) & =\int_{\partial G} \sigma(x) u(x) \bar{\eta}(x) d S_{x}, \quad l_{4}(\eta)=\int_{\partial G} \mu(x) \bar{\eta}(x) d S_{x}, \\
l_{5}(\eta) & =-\int_{G} f(x) \bar{\eta}(x) d x .
\end{aligned}
$$

Because $a, b_{i} \in L^{\infty}(G), f \in L^{2}(G), u, \frac{\partial u}{\partial x_{i}} \in L^{2}(G)$ for any $i=1,2, \ldots, n$, boundedness of functionals $l_{1, u}, l_{2, u}, l_{5}$ follows from the Cauchy-Bunyakovski inequality.

Let us prove boundedness of the functional $l_{3, u}$. Using the Cauchy-Bunyakovski inequality, condition (2.6), the trace description for domains of class $O P_{\varphi}$ [14] and boundedness of $I_{2}$ we obtain the following inequality:

$$
\begin{array}{r}
\left|l_{3, u}(\eta)\right|=\left|\int_{\partial G} \sigma u \bar{\eta} d S_{x}\right|=\left|\int_{\partial G} \frac{\sigma}{\varphi} \varphi u \bar{\eta} d S_{x}\right| \leq M_{\sigma}\|u\|_{2, \varphi, \partial G} \cdot\|\eta\|_{2, \varphi, \partial G} \\
\leq M_{\sigma}\left\|I_{2}\right\|^{2}\|u\|_{W_{2}^{1}(G)}\|\eta\|_{W_{2}^{1}(G)} .
\end{array}
$$

In (3.3) the $W_{2}^{1}(G)$-norm of functions is induced by the inner product (2.7). Constants $M_{\sigma}$ and $\left\|I_{2}\right\|$ depend only on $G$ and function $\sigma$. Therefore functional $l_{3, u}$ is bounded. A similar argument is correct for $l_{4}$ :

$$
\begin{array}{r}
\left|l_{4}(\eta)\right|=\left|\int_{\partial G} \mu \bar{\eta} d S_{x}\right|=\left|\int_{\partial G} \frac{\mu}{\varphi} \varphi \bar{\eta} d S_{x}\right| \leq\left\|\frac{\mu}{\varphi}\right\|_{L^{2, \varphi}(\partial G)} \cdot\|\eta\|_{W_{2}^{1}(G)} \\
\leq\left\|I_{2}\right\|\|\mu\|_{L^{2, \frac{1}{\varphi}(\partial G)}} \cdot\|\eta\|_{W_{2}^{1}(G)} .
\end{array}
$$


We will use the notation $C, C_{1}, C_{2}, \ldots$ for different positive constants.

By the Riess theorem there exist such bounded operators $B_{i}: W_{2}^{1}(G) \rightarrow W_{2}^{1}(G)$, $i=1,2,3$ that $l_{i, u}(\eta):=\left[B_{i} u, \eta\right], l_{4}(\eta):=\left[B_{4} \mu, \eta\right], l_{5}(\eta):=\left[B_{5} f, \eta\right], \eta \in W_{2}^{1}(G)$.

Denote $F:=B_{4} \mu+B_{5} f$. Rewrite (2.4) using $B_{i}$ and $F$ as

$$
\left[u+\left(B_{1}+B_{2}+B_{3}\right) u, \eta\right]=[F, \eta] .
$$

We will also use a short notation $A:=B_{1}+B_{2}+B_{3}$.

Let us prove compactness of operator $A$. The operator $A$ is compact if operators $B_{1}, B_{2}, B_{3}$ are compact. It is enough to prove that for any operator $B_{i}, i=$ $1,2,3$, an image of a weakly convergent sequence contains a strongly convergent subsequence.

Let a sequence $\left\{u_{k}\right\}_{1}^{\infty}$ weakly converge to $u_{0}$ in $W_{2}^{1}(G)$. By continuity of $B_{i}$ the sequence $B_{i} u_{k}$ weakly converges to $B_{i} u_{0}$. By compactness of operators $I_{1}$ and $I_{2}$ we can suppose (without loss of generality) that sequences $\left\{u_{k}\right\}_{1}^{\infty}$ and $\left\{B_{i} u_{k}\right\}_{1}^{\infty}$ strongly converge in $L^{2}(G)$ and $L^{2, \varphi}(\partial G)$ correspondingly.

Let us start from $B_{1}$. For simplicity we will use short notations $l_{i}, i=1,2,3$, instead of $l_{i, u_{k}-u_{m}}$. By the definition of $B_{1}$,

$$
\begin{gathered}
{\left[B_{1}\left(u_{k}-u_{m}\right), B_{1}\left(u_{k}-u_{m}\right)\right]=\left|l_{1}\left(B_{1}\left(u_{k}-u_{m}\right)\right)\right|} \\
\leq M\left\|B_{1} u_{k}-B_{1} u_{m}\right\|_{L^{2}(G)} .
\end{gathered}
$$

Here $M$ is a positive constant. Because $[v, v] \sim\|v\|_{W_{2}^{1}(G)}^{2}$ we have

$$
\left\|B_{1} u_{k}-B_{1} u_{m}\right\|_{W_{2}^{1}(G)}^{2} \leq C\left\|B_{1} u_{k}-B_{1} u_{m}\right\|_{L^{2}(G)}
$$

for a positive constant $C$.

The last inequality means that $\left\{B_{1} u_{k}\right\}_{1}^{\infty}$ strongly converges to $B_{1} u_{0}$ in $W_{2}^{1}(G)$. Therefore operator $B_{1}$ is compact.

A similar argument is correct for operator $B_{2}$ :

$$
\begin{gathered}
\left\|B_{2}\left(u_{k}-u_{m}\right)\right\|_{W_{2}^{1}(G)}^{2} \sim\left[B_{2}\left(u_{k}-u_{m}\right), B_{2}\left(u_{k}-u_{m}\right)\right] \\
=\left|l_{2}\left(B_{2}\left(u_{k}-u_{m}\right)\right)\right| \leq C\left\|B_{2} u_{k}-B_{2} u_{m}\right\|_{L^{2}(G)}
\end{gathered}
$$

for a positive constant $C$. Therefore sequence $\left\{B_{2} u_{k}\right\}_{1}^{\infty}$ strongly converges to $B_{2} u_{0}$ in $W_{2}^{1}(G)$ and operator $B_{2}$ is compact.

For operator $B_{3}$ we will use compactness of $I_{2}$. Using similar arguments we have

$$
\begin{gathered}
\left\|B_{3}\left(u_{k}-u_{m}\right)\right\|_{W_{2}^{1}(G)}^{2} \sim\left[B_{3}\left(u_{k}-u_{m}\right), B_{3}\left(u_{k}-u_{m}\right)\right], \\
=\left|l_{3}\left(B_{3}\left(u_{k}-u_{m}\right)\right)\right| \leq M_{\sigma}\left\|u_{k}-u_{m}\right\|_{L^{2, \varphi}(\partial G)} \cdot\left\|B_{3}\left(u_{k}-u_{m}\right)\right\|_{L^{2, \varphi}(\partial G)},
\end{gathered}
$$

proving compactness of $B_{3}$.

Hence operator $A=B_{1}+B_{2}+B_{3}$ is compact. Therefore the operator $I+A$ is of the Fredholm type.

\section{Compactness of embedding operator $I_{p}: W_{p}^{1}(G) \rightarrow L^{p, \varphi}(\partial G)$}

Theorem 2.3 is a special case for $p=2$ of the following result.

Theorem 4.1. If $G \in O P_{\varphi}$, then the embedding operator $I_{p}: W_{p}^{1}(G) \rightarrow L^{p, \varphi}(\partial G)$ is compact.

Fix a domain $G \in O P_{\varphi}$. Without loss of generality we can suppose that $0<$ $\varphi^{\prime}\left(x_{n}\right)<1$ for any $0 \leq x_{n} \leq 1$, a top of peak $O$ coincides with the origin of 
coordinates and there exists such a neighborhood $V(O)$ of $O$ that $V(O) \cap G$ can be represented as

$$
\left\{x=\left(x^{\prime}, x_{n}\right) \in \mathbb{R}^{n}: 0<x_{n}<1,\left|x^{\prime}\right|<\varphi\left(x_{n}\right)\right\} .
$$

Here notice that $x=\left(x^{\prime}, x_{n}\right) \in \mathbb{R}^{n}, x^{\prime} \in \mathbb{R}^{n-1}, x_{n} \in \mathbb{R}$.

For the proof of Theorem 4.1 we need the following technical result:

Lemma 4.2. Any function $F \in W_{p}^{1}(G), 1<p<\infty$, has the following representation:

$$
F(x)=\alpha\left(x_{n}\right)+R(x),
$$

where $\alpha \in W_{p}^{1}(G)$ is a function of one variable, $R \in W_{p}^{1}(G)$, and the following inequality is correct:

$$
\left\|\varphi^{-1}\left(x_{n}\right) R\right\|_{L^{p}(G)} \leq C\|F\|_{W_{p}^{1}(G)}<\infty
$$

for some constant $C$ that depends only on $G$ and $n$.

For the reader's convenience we give here a complete, comparatively simple and independent proof of this result that was proved by the second author in [14].

Proof. Choose nonnegative functions $h \in C_{0}^{\infty}\left(\mathbb{R}^{n-1}\right)$ and $K \in C_{0}^{\infty}(\mathbb{R})$ such that $\operatorname{supp} h \subset B_{1}\left(0^{\prime}\right):=\left\{x^{\prime}=\left(x_{1}, \ldots, x_{n-1}\right) \in \mathbb{R}^{n-1}:\left|x^{\prime}\right|<1\right\}, \operatorname{supp} K \subset\left[\frac{1}{2}, 1\right]$,

$$
\begin{aligned}
\int_{\mathbb{R}^{n-1}} h\left(x^{\prime}\right) d x^{\prime} & =1, \int_{-\infty}^{\infty} K(t) d t=1 . \text { Suppose } \\
& \Omega\left(x, r, \theta^{\prime}, y\right)=\varphi^{-n}\left(x_{n}\right) h\left(\frac{x^{\prime}+r \theta^{\prime}}{\varphi\left(x_{n}\right)}\right) r^{n-2} K\left(\frac{y}{\varphi\left(x_{n}\right)}\right),
\end{aligned}
$$

where $r \geq 0, y \in \mathbb{R}^{1}$, and $\theta^{\prime} \in S_{1}\left(0^{\prime}\right)=\partial B_{1}\left(0^{\prime}\right)$. We will use a family of kernels

$$
\Omega_{\varepsilon}:=\Omega\left(x, \frac{r}{\varepsilon}, \theta^{\prime}, \frac{y}{\varepsilon}\right) .
$$

Denote

$$
F_{\varepsilon}(x)=\int_{S_{1}\left(0^{\prime}\right)} d S_{\theta^{\prime}} \int_{0}^{\infty} d r \int_{-\infty}^{\infty} F\left(x^{\prime}+r \theta^{\prime}, x_{n}+y\right) \Omega\left(x, \frac{r}{\varepsilon}, \theta^{\prime}, \frac{y}{\varepsilon}\right) \frac{d y}{\varepsilon^{2}} .
$$

The proof of this lemma can be divided onto four parts.

1. Let us demonstrate that $F_{\epsilon} \rightarrow F$ in $L^{p, l o c}(G)$ for $\epsilon \rightarrow 0$. Using the change of variables $r \vartheta^{\prime}=z^{\prime}, y=z_{n}$ we obtain that

$$
F_{\varepsilon}(x)=\int_{R^{n}} F(x+z) h\left(\frac{\varepsilon x^{\prime}+z^{\prime}}{\varepsilon \varphi\left(x_{n}\right)}\right) K\left(\frac{z_{n}}{\varepsilon \varphi\left(x_{n}\right)}\right) \frac{d z}{\varepsilon^{n} \varphi^{n}\left(x_{n}\right)} .
$$

Let $U$ be a compact subset of $G$. Denote $x_{n}^{0}=\inf \left\{x_{n}: x=\left(x^{\prime}, x_{n}\right) \in U\right\}$. Then $x_{n}^{0}>0$. Using monotonicity of $\varphi$ and compactness of $U$ we have that $\varphi\left(x_{n}^{0}\right)<$ $\varphi\left(x_{n}\right)<C_{0} \varphi\left(x_{n}^{0}\right)$, where $x=\left(x^{\prime}, x_{n}\right) \in U$ and the constant $C_{0}$ depends only on $U$ and $\varphi$. By construction of $K$ we have $\frac{1}{2} \leq \frac{z_{n}}{\varepsilon \varphi\left(x_{n}\right)} \leq 1$. Hence

$$
K\left(\frac{z_{n}}{\varepsilon \varphi\left(x_{n}\right)}\right) \leq C_{1} \chi\left(\frac{z_{n}}{C_{0} \varepsilon \varphi\left(x_{n}^{0}\right)}\right)
$$


Because $h\left(\frac{\varepsilon x^{\prime}+z^{\prime}}{\varepsilon \varphi\left(x_{n}\right)}\right) \neq 0$ for $\left|\frac{\varepsilon x^{\prime}+z^{\prime}}{\varepsilon \varphi\left(x_{n}\right)}\right|<1$, then

$$
\left|h\left(\frac{\varepsilon x^{\prime}+z^{\prime}}{\varepsilon \varphi\left(x_{n}\right)}\right)\right| \leq C_{2} \chi\left(\frac{\left|z^{\prime}\right|}{2 C_{0} \varepsilon \varphi\left(x_{n}^{0}\right)}\right) .
$$

In these inequalities, $C_{1}=\sup \{K(\tau): \tau \in \mathbf{R}\}, C_{2}=\sup \left\{\left|h\left(u^{\prime}\right)\right|: u^{\prime} \in \mathbf{R}^{\mathbf{n}-\mathbf{1}}\right\}$ and $\chi$ is the indicator of $[0 ; 1]$. The second inequality is based on the inequality $\left|z^{\prime}\right| \leq \varepsilon\left|x^{\prime}\right|+\varepsilon \varphi\left(x_{n}\right) \leq 2 \varepsilon \varphi\left(x_{n}\right) \leq 2 C_{0} \varepsilon \varphi\left(x_{n}^{0}\right)$ that is a direct consequence of an obvious inequality $\left|\varepsilon x^{\prime}+z^{\prime}\right| \leq \varepsilon \varphi\left(x_{n}\right)$.

Because

$$
\int_{\mathbf{R}^{\mathbf{n}}} h\left(\frac{\varepsilon x^{\prime}+z^{\prime}}{\varepsilon \varphi\left(x_{n}\right)}\right) K\left(\frac{z_{n}}{\varepsilon \varphi\left(x_{n}\right)}\right) \frac{d z}{\varepsilon^{n} \varphi^{n}\left(x_{n}\right)}=1,
$$

we get

$$
\left|F_{\varepsilon}(x)-F(x)\right| \leq C \int_{\mathbf{R}^{\mathbf{n}}}|F(x+z)-F(x)| \chi\left(\frac{z_{n}}{C_{0} \varepsilon \varphi\left(x_{n}^{0}\right)}\right) \chi\left(\frac{\left|z^{\prime}\right|}{2 C_{0} \varepsilon \varphi\left(x_{n}^{0}\right)}\right) \frac{d z}{\varepsilon^{n} \varphi^{n}\left(x_{n}^{0}\right)} .
$$

Integrating both parts of the previous inequality in degree $p$ on $U$ and using the Minkovski inequality

$$
\begin{aligned}
& \left\|F_{\varepsilon}-F\right\|_{L^{p}(U)} \leq \\
& C \int_{R^{n}}\left(\int_{U}|F(x+z)-F(x)|^{p} d x\right)^{\frac{1}{p}} \chi\left(\frac{z_{n}}{C_{0} \varepsilon \varphi\left(x_{n}^{0}\right)}\right) \chi\left(\frac{\left|z^{\prime}\right|}{2 C_{0} \varepsilon \varphi\left(x_{n}^{0}\right)}\right) \frac{d z}{\varepsilon^{n} \varphi^{n}\left(x_{n}^{0}\right)} \\
& \leq C \sup \left\{\|F(x+z)-F(x)\|_{L^{p}(U)}:|z| \leq 2 C_{0} \varepsilon \varphi\left(x_{n}^{0}\right)\right\} .
\end{aligned}
$$

Using continuity of function $F \in L^{p}(G)$ in the sense of $L^{p}$ (see [13, ch.1) the proof can be finished.

Therefore we obtained the following integral representation:

$$
F(x)=F_{1}(x)-\int_{0}^{1} \frac{\partial}{\partial \varepsilon} F_{\varepsilon}(x) d \varepsilon
$$

for any $F \in L^{p}(G)$.

We will use the short notation

$$
R(x)=-\int_{0}^{1} \frac{\partial}{\partial \varepsilon} F_{\varepsilon}(x) d \varepsilon
$$

Recall that

$$
F_{1}(x)=\int_{-\infty}^{\infty} d y \int_{S} d S_{\theta}^{\prime} \int_{0}^{\infty} F\left(x^{\prime}+r \theta^{\prime}, x_{n}+y\right) \Omega\left(x, r, \theta^{\prime}, y\right) d r .
$$

Using the following change of variables $x^{\prime}+r \theta^{\prime}=z^{\prime}, x_{n}+y=z_{n}, r^{n-2} d r d \theta^{\prime}=$ $d z^{\prime}, d y=d z_{n}$ we obtain

$$
\alpha\left(x_{n}\right):=F_{1}(x)=\int_{R^{n}} F(z) h\left(\frac{z^{\prime}}{\varphi\left(x_{n}\right)}\right) K\left(\frac{z_{n}-x_{n}}{\varphi\left(x_{n}\right)}\right) \frac{d z}{\varphi^{n}\left(x_{n}\right)} .
$$

Here we used a new notation $\alpha\left(x_{n}\right):=F_{1}(x)$ emphasizing that function $F_{1}(x)$ depends only on $x_{n}$. 
2. Let us demonstrate that $\alpha \in W_{p}^{1}(G)$ for $1<p<\infty$. By construction of $\alpha$ we have

$$
\begin{aligned}
\| \alpha & \|_{L^{p}(G)}^{p}=\int_{G}\left|\alpha\left(x_{n}\right)\right|^{p} d x=\int_{0}^{1} d x_{n} \int_{\left\{\left|x^{\prime}\right|<\varphi\left(x_{n}\right)\right\}}\left|\alpha\left(x_{n}\right)\right|^{p} d x^{\prime} \\
& \leq \int_{0}^{1} \int_{\left\{\left|x^{\prime}\right|<\varphi\left(x_{n}\right)\right\}}\left|\int_{R^{n}}\right| F(z)\left|\cdot h\left(\frac{z^{\prime}}{\varphi\left(x_{n}\right)}\right) \cdot K\left(\frac{z_{n}-x_{n}}{\varphi\left(x_{n}\right)}\right) \frac{d z}{\varphi^{n}\left(x_{n}\right)}\right|^{p} d x^{\prime} d x_{n} .
\end{aligned}
$$

Recall that supph $\subset B_{1}\left(0^{\prime}\right)$ and $K \subset[1 / 2 ; 1]$. Therefore the domain of integration for $z$ is the following set: $U:=\left\{z:\left|z^{\prime}\right|<\varphi\left(x_{n}\right), x_{n}+\varphi\left(x_{n}\right) / 2<z_{n}<x_{n}+\varphi\left(x_{n}\right)\right\}$, which belongs to $G$.

There exist $\delta>0$ and $o<c<x_{n}$ such that

$$
\varphi\left(x_{n}+\delta \varphi\left(x_{n}\right)\right)=\varphi\left(x_{n}\right)+\varphi^{\prime}(c) \delta \varphi\left(x_{n}\right)=\varphi\left(x_{n}\right)\left(1+\delta \varphi^{\prime}(c)\right) .
$$

By monotonicity of $\varphi$ we have for $\delta=1 / 2$ the following inequality:

$$
\frac{\varphi\left(x_{n}\right)}{2} \leq \varphi\left(z_{n}\right) \leq \frac{3 \varphi\left(x_{n}\right)}{2} .
$$

The integrand in (4.4) can be rewritten in a more convenient way as

$$
\varphi^{-n}|F| \cdot|h| \cdot|K|=|F| \cdot\left(|h|^{\frac{1}{p}}|K|^{\frac{1}{p}} \varphi^{-\frac{n}{p}}\right) \cdot\left(|h|^{\frac{p-1}{p}}|K|^{\frac{p-1}{p}} \varphi^{-\frac{(p-1) n}{p}}\right) .
$$

Using the Hölder inequality and changing the order of integration we obtain

$$
\|\alpha\|_{L_{p}(G)}^{p}=C \int_{G}|F(z)|^{p} \Psi\left(z, x_{n}\right) d z
$$

where

$$
\begin{gathered}
C=\left\{\int_{G} \Psi\left(z, x_{n}\right) d z\right\}^{p-1} \\
\Psi\left(z, x_{n}\right)=\int_{0}^{1} d x_{n} \int_{\left\{\left|x^{\prime}\right|<\varphi\left(x_{n}\right)\right\}} h\left(\frac{z^{\prime}}{\varphi\left(x_{n}\right)}\right) K\left(\frac{z_{n}-x_{n}}{\varphi\left(x_{n}\right)}\right) \frac{d x^{\prime}}{\varphi^{n}\left(x_{n}\right)} .
\end{gathered}
$$

We will estimate $\Psi\left(z, x_{n}\right)$.

Let us first change $x_{n}$ into $\tau:=\frac{z_{n}-x_{n}}{\varphi\left(x_{n}\right)}$. Hence

$$
\frac{d x_{n}}{\varphi\left(x_{n}\right)}=-\frac{d \tau}{1+\frac{z_{n}-x_{n}(\tau)}{\varphi\left(x_{n}(\tau)\right)} \varphi^{\prime}\left(x_{n}\right)} .
$$

Therefore

$$
\int_{0}^{1} K\left(\frac{z_{n}-x_{n}}{\varphi\left(x_{n}\right)}\right) \frac{d x_{n}}{\varphi\left(x_{n}\right)} \leq C \int_{-\infty}^{\infty} K(\tau) \frac{d \tau}{1-\varphi^{\prime}\left(x_{n}(\tau)\right)}<\infty
$$

because of the boundedness of function $K$ with compact support. Using the same argument for (4.6) we obtain finally the following estimate for the $L^{p}$-norm of $\alpha$ :

$$
\|\alpha\|_{L^{p}(G)}^{p} \leq C \int_{G}|F(z)|^{p} d z
$$

Let us prove that $\alpha \in W_{p}^{1}(G)$. 
Using the following change of variables in (4.3),

$$
z^{\prime}=\varphi\left(x_{n}\right) s^{\prime}, z_{n}=x_{n}+\varphi\left(x_{n}\right) s_{n}
$$

we get

$$
\alpha\left(x_{n}\right)=\int_{R^{n}} F\left(\varphi\left(x_{n}\right) s^{\prime}, x_{n}+\varphi\left(x_{n}\right) s_{n}\right) h\left(s^{\prime}\right) K\left(s_{n}\right) d s .
$$

After differentiation we obtain

$$
\begin{aligned}
\alpha^{\prime}\left(x_{n}\right)=\int_{R^{n}}\left(\varphi^{\prime}\left(\nabla^{\prime} F\left(\varphi\left(x_{n}\right) s^{\prime}, x_{n}+\varphi\left(x_{n}\right) s_{n}\right), s^{\prime}\right)\right. \\
\left.\quad+D_{n} F\left(\varphi\left(x_{n}\right) s^{\prime}, x_{n}+\varphi\left(x_{n}\right) s_{n}\right)\left(1+\varphi^{\prime}\left(x_{n}\right) s_{n}\right)\right) h\left(s^{\prime}\right) K\left(s_{n}\right) d s .
\end{aligned}
$$

Returning to the original variable we finally get

$$
\begin{aligned}
\left|\alpha^{\prime}\left(x_{n}\right)\right| \leq C \int_{R^{n}}\left|\nabla^{\prime} F(z)\right| & \cdot h\left(\frac{z^{\prime}}{\varphi\left(x_{n}\right)}\right) \cdot K\left(\frac{z_{n}-x_{n}}{\varphi\left(x_{n}\right)}\right) \frac{d z}{\varphi^{n}\left(x_{n}\right)} \\
& +C \int_{R^{n}}\left|D_{n} F(z)\right| \cdot h\left(\frac{z^{\prime}}{\varphi\left(x_{n}\right)}\right) \cdot K\left(\frac{z_{n}-x_{n}}{\varphi\left(x_{n}\right)}\right) \frac{d z}{\varphi^{n}\left(x_{n}\right)} .
\end{aligned}
$$

This inequality permits us to estimate $\left\|\alpha^{\prime}\right\|_{L^{p}(G)}$ by the same way as $\|\alpha\|_{L^{p}(G)}$. Therefore $\alpha \in W_{p}^{1}(G)$.

3. Function $R$ can be represented as $R=R_{1}+R_{2}$, where

$$
\begin{aligned}
& R_{1}(x)=-\int_{0}^{1} \frac{d \varepsilon}{\varepsilon^{n+1}} \int_{\mathbb{R}^{n}}\left(\nabla^{\prime} F(z), z^{\prime}-x^{\prime}\right) h\left(\frac{z^{\prime}-(1-\varepsilon) x^{\prime}}{\varepsilon \varphi\left(x_{n}\right)}\right) K\left(\frac{z_{n}-x_{n}}{\varepsilon \varphi\left(x_{n}\right)}\right) \frac{d z}{\varphi^{n}\left(x_{n}\right)}, \\
& R_{2}(x)=-\int_{0}^{1} \frac{d \varepsilon}{\varepsilon^{n+1}} \int_{\mathbb{R}^{n}} D_{n} F(z) \cdot\left(z_{n}-x_{n}\right) K\left(\frac{z_{n}-x_{n}}{\varepsilon \varphi\left(x_{n}\right)}\right) h\left(\frac{z^{\prime}-(1-\varepsilon) x^{\prime}}{\varepsilon \varphi\left(x_{n}\right)}\right) \frac{d z}{\varphi^{n}\left(x_{n}\right)} .
\end{aligned}
$$

Here $\nabla^{\prime}=\left(\frac{\partial}{\partial z_{1}}, \ldots, \frac{\partial}{\partial z_{n-1}}\right), z=\left(z^{\prime}, z_{n}\right), D_{n}=\frac{\partial}{\partial z_{n}}$.

Let us verify this representation. Remember first that

$$
\Omega\left(x, \frac{r}{\epsilon}, \theta^{\prime}, \frac{y}{\epsilon}\right)=\frac{r^{n-2}}{\varphi^{n}\left(x_{n}\right) \epsilon^{n-2}} h\left(\frac{\epsilon x^{\prime}+r \theta^{\prime}}{\epsilon \varphi\left(x_{n}\right)}\right) K\left(\frac{y}{\epsilon \varphi\left(x_{n}\right)}\right) .
$$

Hence

$$
\begin{aligned}
\frac{\partial}{\partial \varepsilon} & {\left[\varepsilon^{-2} \Omega\left(x, \frac{r}{\varepsilon}, \theta^{\prime}, \frac{y}{\varepsilon}\right)\right] } \\
& =-\frac{n r^{n-2}}{\varepsilon^{n+1} \varphi^{n}\left(x_{n}\right)} h K-\frac{r^{n-1}}{\varepsilon^{n+2} \varphi^{n+1}\left(x_{n}\right)} \sum_{j=1}^{n-1} D_{j} h \theta_{j}-\frac{y r^{n-2}}{\varepsilon^{n+2} \varphi^{n+1}\left(x_{n}\right)} h K^{\prime} \\
& -\frac{K}{\varepsilon^{n+1} \varphi^{n}\left(x_{n}\right)} \frac{\partial}{\partial r}\left[r^{n-1} h\right]-\frac{r^{n-2}}{\varepsilon^{n+1} \varphi^{n}\left(x_{n}\right)} h \frac{\partial}{\partial y}\left[y K\left(\frac{y}{\varepsilon \varphi\left(x_{n}\right)}\right)\right] .
\end{aligned}
$$


Using (4.7) we get the following expression for $R(x)$ :

$$
\begin{aligned}
R(x)= & -\int_{0}^{1} \frac{\partial F_{\epsilon}}{\partial \epsilon} d \epsilon \\
= & -\int_{0}^{1} d \epsilon \int_{-\infty}^{\infty} d y \int_{S} d S_{\theta}^{\prime} \int_{0}^{\infty} F\left(x^{\prime}+r \theta^{\prime}, x_{n}+y\right) \frac{\partial}{\partial \epsilon}\left[\Omega\left(x^{\prime}, \frac{r}{\epsilon}, \theta^{\prime}, \frac{y}{\epsilon}\right) \epsilon^{-2}\right] d r \\
= & -\int_{0}^{1} d \varepsilon \int_{S} d S_{\theta^{\prime}} \int_{0}^{\infty}\left(\sum_{j=1}^{n-1} D_{j} F \theta_{j}\right) r^{n-1} h\left(\frac{\varepsilon x^{\prime}+r \theta^{\prime}}{\varepsilon \varphi\left(x_{n}\right)}\right) d r \\
& =\int_{0}^{1} d \varepsilon \int_{S} d S_{\theta^{\prime}} \int_{0}^{\infty} h\left(\frac{\varepsilon x^{\prime}+r \theta^{\prime}}{\varepsilon \varphi\left(x_{n}\right)}\right) \frac{r^{n-2}}{\varepsilon^{n+1} \varphi^{n}\left(x_{n}\right)} d r \int_{-\infty}^{\infty} y D_{n} F K\left(\frac{y}{\varepsilon \varphi\left(x_{n}\right)}\right) d y .
\end{aligned}
$$

Using the following change of variables,

$$
z^{\prime}=x^{\prime}+r \theta^{\prime}, d z^{\prime}=r^{n-2} d r d S_{\theta}^{\prime}, x_{n}+y=z_{n}, d z_{n}=d y,
$$

we obtain the above-claimed decomposition $R=R_{1}+R_{2}$ :

$$
\begin{aligned}
R(x)= & -\int_{0}^{1} \frac{d \epsilon}{\epsilon^{n+1} \varphi^{n}\left(x_{n}\right)} \int_{R^{n}}\left(\nabla^{\prime} F(z), z^{\prime}-x^{\prime}\right) K\left(\frac{z_{n}-x_{n}}{\epsilon \varphi\left(x_{n}\right)}\right) h\left(\frac{z^{\prime}-(1-\epsilon) x^{\prime}}{\epsilon \varphi\left(x_{n}\right)}\right) d z \\
& -\int_{0}^{1} \frac{d \epsilon}{\epsilon^{n+1} \varphi^{n}\left(x_{n}\right)} \int_{R^{n}} D_{n} F(z) \cdot\left(z_{n}-x_{n}\right) K\left(\frac{z_{n}-x_{n}}{\epsilon \varphi\left(x_{n}\right)}\right) h\left(\frac{z^{\prime}-(1-\epsilon) x^{\prime}}{\epsilon \varphi\left(x_{n}\right)}\right) d z \\
= & R_{1}+R_{2} .
\end{aligned}
$$

4. Similarly to the plane case 15 and to the proof of (4.2) we will demonstrate that $\varphi^{-1} R_{i} \in L^{p}(G), i=1,2$. We will prove this fact only for $R_{1}$. For $R_{2}$ the proof is similar.

We have

$$
\begin{aligned}
& \left|\varphi^{-1}\left(x_{n}\right) R_{1}(x)\right| \\
\leq & \int_{0}^{1} d \varepsilon \int_{\mathbb{R}^{n}}\left|\nabla^{\prime} F(z)\right| \cdot\left|\frac{z^{\prime}-x^{\prime}}{\varepsilon \varphi\left(x_{n}\right)}\right| h\left(\frac{z^{\prime}-(1-\varepsilon) x^{\prime}}{\varepsilon \varphi\left(x_{n}\right)}\right) K\left(\frac{z_{n}-x_{n}}{\varepsilon \varphi\left(x_{n}\right)}\right) \frac{d z}{\varepsilon^{n} \varphi^{n}\left(x_{n}\right)} .
\end{aligned}
$$

Denote by $X(x)$ the extension of $\left|\varphi^{-1}\left(x_{n}\right) R_{1}(x)\right|$ on $\mathbb{R}^{n} \backslash G$ by zero and by $Y(z)$ the extension of $\left|\nabla^{\prime} F(z)\right|$ by zero on $\mathbb{R}^{n} \backslash G$. Recall the generalized Minkovski inequality. Let $\psi(x, y)$ be a measurable nonnegative function defined on $A \times B$, where $A \in R^{n}, B \in R^{m}$ are measurable sets. Then for any $1 \leq p<\infty$,

$$
\left\{\int_{A}\left(\int_{B} \psi(x, y) d y\right)^{p} d x\right\}^{1 / p} \leq \int_{B}\left(\int_{A} \psi^{p}(x, y) d x\right)^{1 / p} d y
$$


Using the generalized Minkovski inequality we get

$$
\begin{aligned}
\|X\|_{L_{p}\left(\mathbf{R}^{n}\right)} \leq & \int_{0}^{1}\left\{\int _ { \mathbf { R } ^ { n } } \left[\frac{1}{\varepsilon^{n} \varphi^{n}\left(x_{n}\right)} \int_{\mathbf{R}} \Phi(z, x)\right.\right. \\
& \left.\left.\left(\int_{\mathbf{R}^{n-1}} h\left(\frac{z^{\prime}-(1-\varepsilon) x^{\prime}}{\varepsilon \varphi\left(x_{n}\right)}\right) d z^{\prime}\right)^{\frac{p-1}{p}} K d z_{n}\right]^{p} d x\right\}^{1 / p} d \varepsilon .
\end{aligned}
$$

Here

Because

$$
\Phi(z, x)=\left\{\int_{\mathbf{R}^{n-1}} Y^{p}(z) h\left(\frac{z^{\prime}-(1-\varepsilon) x^{\prime}}{\varepsilon \varphi\left(x_{n}\right)}\right) d z^{\prime}\right\}^{1 / p} .
$$

$$
\left(\int_{\mathbf{R}^{n-1}} h\left(\frac{z^{\prime}-(1-\varepsilon) x^{\prime}}{\varepsilon \varphi\left(x_{n}\right)}\right) d z^{\prime}\right)^{\frac{p-1}{p}} \leq C\left[\varepsilon \varphi\left(x_{n}\right)\right]^{\frac{(n-1)(p-1)}{p}}
$$

we finally obtain

$$
\|X\|_{L_{p}\left(\mathbf{R}^{n}\right)} \leq C \int_{0}^{1}\left\{\int_{\mathbf{R}^{n}}\left[\int_{\mathbf{R}} \Phi(z, x) K\left(\frac{z_{n}-x_{n}^{\prime}}{\varepsilon \varphi\left(x_{n}\right)}\right) d z_{n}\right]^{p} \frac{d x}{\left(\varepsilon \varphi\left(x_{n}\right)\right)^{n+p-1}}\right\}^{1 / p} d \varepsilon .
$$

Using for the term in square brackets from the right hand side of (4.10) the Hölder inequality for the variable $z_{n}$ and taking into account the inequality

$$
\left\{\int_{-\infty}^{\infty} K\left(\frac{z_{n}-x_{n}}{\varepsilon \varphi\left(x_{n}\right)}\right) d z_{n}\right\}^{p-1} \leq C\left(\varepsilon \varphi\left(x_{n}\right)\right)^{p-1}
$$

we obtain the following $L^{p}$-estimate:

$$
\begin{aligned}
& \|X\|_{L^{p}(G)} \leq \\
& C \int_{0}^{1}\left\{\int_{\mathbb{R}^{n}} Y^{p}(z)\left[\int_{\mathbb{R}^{n}} h\left(\frac{z^{\prime}-(1-\varepsilon) x^{\prime}}{\varepsilon \varphi\left(x_{n}\right)}\right) K\left(\frac{z_{n}-x_{n}}{\varepsilon \varphi\left(x_{n}\right)}\right) \frac{d x}{\left(\varepsilon \varphi\left(x_{n}\right)\right)^{n}}\right] d z\right\}^{\frac{1}{p}} d \varepsilon .
\end{aligned}
$$

Denote by $J$ the term in square brackets on the right hand side of (4.11). By direct calculations we have

$$
\begin{aligned}
J=\int_{\mathbb{R}^{n}} h\left(\frac{z^{\prime}-(1-\varepsilon) x^{\prime}}{\varepsilon \varphi\left(x_{n}\right)}\right) K\left(\frac{z_{n}-x_{n}}{\varepsilon \varphi\left(x_{n}\right)}\right) \frac{d x}{\left(\varepsilon \varphi\left(x_{n}\right)\right)^{n}} \\
=\int_{-\infty}^{\infty} K\left(\frac{z_{n}-x_{n}}{\varepsilon \varphi\left(x_{n}\right)}\right)\left(\int_{\mathbb{R}^{n-1}} h\left(\frac{z^{\prime}-(1-\varepsilon) x^{\prime}}{\varepsilon \varphi\left(x_{n}\right)}\right) d x^{\prime}\right) \frac{d x_{n}}{\left(\varepsilon \varphi\left(x_{n}\right)\right)^{n}} \\
\leq C \int_{-\infty}^{\infty} K\left(\frac{z_{n}-x_{n}}{\varepsilon \varphi\left(x_{n}\right)}\right) \frac{d x_{n}}{\varepsilon \varphi\left(x_{n}\right)} .
\end{aligned}
$$

For the last inequality we used the following estimate:

$$
\int_{\mathbf{R}^{n-1}} h\left(\frac{z^{\prime}-(1-\varepsilon) x^{\prime}}{\varepsilon \varphi\left(x_{n}\right)}\right) d x^{\prime} \leq C\left[\varepsilon \varphi\left(x_{n}\right)\right]^{n-1} .
$$


This estimate can be proved by the following way. Let

$$
A=\left\{x^{\prime} \in \mathbf{R}^{n-1}:\left|\frac{z^{\prime}-x^{\prime}}{\varepsilon \varphi\left(x_{n}\right)}\right|<1\right\}, B=\left\{x^{\prime} \in \mathbf{R}^{n-1}:\left|z^{\prime}-x^{\prime}\right|<2 \varepsilon \varphi\left(x_{n}\right)\right\} .
$$

Using the definition of class $O P_{\varphi}$ and the inequalities

$$
\left|\frac{z^{\prime}-x^{\prime}}{\varepsilon \varphi\left(x_{n}\right)}\right|-1 \leq\left|\frac{z^{\prime}-x^{\prime}}{\varepsilon \varphi\left(x_{n}\right)}\right|-\frac{\left|x^{\prime}\right|}{\varphi\left(x_{n}\right)} \leq\left|\frac{z^{\prime}-(1-\varepsilon) x^{\prime}}{\varepsilon \varphi\left(x_{n}\right)}\right|<1
$$

it follows that $A \subset B$. Therefore

$$
\int_{\mathbf{R}^{n-1}} h\left(\frac{z^{\prime}-(1-\varepsilon) x^{\prime}}{\varepsilon \varphi\left(x_{n}\right)}\right) d x^{\prime} \leq \int_{A} h d x^{\prime} \leq \int_{B} h d x^{\prime} .
$$

Let us change variable $x_{n}$ to $\tau:=\frac{z_{n}-x_{n}}{\varepsilon \varphi\left(x_{n}\right)}=\tau\left(x_{n} \rightarrow \tau\right)$. Hence

$$
\frac{d x}{\varepsilon \varphi\left(x_{n}\right)}=-\frac{d \tau}{1+\frac{z_{n}-x_{n}(\tau)}{\varepsilon \varphi\left(x_{n}(\tau)\right)} \varphi^{\prime}\left(x_{n}\right)} \text {. }
$$

Therefore

$$
\int_{-\infty}^{\infty} K\left(\frac{z_{n}-x_{n}}{\varepsilon \varphi\left(x_{n}\right)}\right) \frac{d x}{\varepsilon \varphi\left(x_{n}\right)} \leq C \int_{-\infty}^{\infty} K(\tau) \frac{d \tau}{1-\varepsilon \varphi^{\prime}\left(x_{n}(\tau)\right)} \leq C
$$

where constant $C$ depends only on $F(x)$. Using the last inequality (4.11) we obtain finally

$$
\|X\|_{L^{p}\left(\mathbb{R}^{n}\right)} \leq C\|Y\|_{L^{p}\left(\mathbb{R}^{n}\right)} \leq C\|F\|_{W_{p}^{1}(G)} .
$$

Using the previous Lemma 4.2 we will prove Theorem 4.1 .

Proof of Theorem 4.1. Let $\mathfrak{M} \subset W_{p}^{1}(G)$ be a bounded set and $\Lambda=I_{p}(\mathfrak{M}) \subset$ $L^{p, \varphi}(\partial G)$. To prove compactness of $I_{2}: W_{p}^{1}(G) \rightarrow L^{p, \varphi}(\partial G)$ we need to construct for any $\varepsilon>0$ a finite $\varepsilon$-network of $\Lambda$.

By Lemma 4.2 any function $F \in \mathfrak{M}$ can be represented as $F=\alpha+R, \alpha=\alpha\left(x_{n}\right)$. Denote by $\Lambda_{\alpha}$ the set of all functions $\alpha=F-R$ such that $F \in \mathfrak{M}$, and denote by $\Lambda_{R}$ the set of all functions $R$ that correspond to $F \in \mathfrak{M}$. Hence $\Lambda=\Lambda_{\alpha}+\Lambda_{R}$.

Because the embedding operator $I_{1}: W_{p}^{1}(G) \rightarrow L^{p}(G)$ is compact, the set $\Lambda_{\alpha}$ is relatively compact in $L^{p}(G)$. Therefore for any $\varepsilon_{1}>0$ there exists an $\varepsilon_{1}$ network $\beta_{1}, \ldots, \beta_{N}$ of $\Lambda_{\alpha}$ in $L^{p}(G)$. Denote $G_{\delta}=B_{\delta}(0) \cap G$, where $B_{\delta}(0)=$ $\left\{x \in \mathbb{R}^{n}:|x|<\delta\right\}$. Further we suppose that $0<\delta<1$. Using continuity of function $F \in L^{p}(G)$ in the sense of $L^{p}$ (see [13], ch.1), we can find $\delta>0$ such that for any $j=1,2, \ldots, N$ the following inequality is correct:

$$
\left\|\beta_{j}\right\|_{p, G_{\delta}}<\varepsilon_{1} .
$$

Hence for any $\alpha \in \Lambda_{\alpha}$ there exists such a $\beta_{j}$ that

$$
\|\alpha\|_{p, G_{\delta}} \leq\left\|\alpha-\beta_{j}\right\|_{p, G_{\delta}}+\left\|\beta_{j}\right\|_{p, G_{\delta}}<2 \varepsilon_{1}
$$


By construction of the domain $G$ in a neighborhood of its peak with the top $O$ (see (2.5)) we have by direct calculation

$$
\int_{G_{\delta}}\left|\alpha\left(x_{n}\right)\right|^{p} d x=\omega_{n-1} \int_{0}^{\delta}\left|\alpha\left(x_{n}\right)\right|^{p} \varphi^{n-1}\left(x_{n}\right) d x_{n},
$$

where $\omega_{n-1}$ is the volume of the unit $(n-1)$-dimensional ball.

On the other hand we will demonstrate (using (2.5)) that

$$
\int_{\partial G_{\delta}}\left|\alpha\left(x_{n}\right)\right|^{p} \varphi\left(x_{n}\right) d S_{x} \sim \int_{0}^{\delta}\left|\alpha\left(x_{n}\right)\right|^{p} \varphi^{n-1}\left(x_{n}\right) d x_{n} .
$$

The argument is simple. The manifold $\partial G_{\delta} \backslash\{0\}$ is a finite union of charts of the following type:

$$
\begin{aligned}
\partial G_{\delta, \sigma}^{(n-1)}=\{x & =\left(x^{\prime \prime}, x_{n-1}, x_{n}\right): 0<x_{n}<\delta \\
& \left.x_{n-1}=\sqrt{\varphi^{2}\left(x_{n}\right)-\left|x^{\prime \prime}\right|^{2}},\left|x^{\prime \prime}\right|=\left(\sum_{i=1}^{n-2} x_{i}^{2}\right)^{1 / 2}<\sigma \varphi\left(x_{n}\right)\right\},
\end{aligned}
$$

where $\sigma \in(0,1)$. By direct calculations,

$$
d S_{x}=\varphi\left(x_{n}\right) \sqrt{\frac{1+\left(\varphi^{\prime}\left(x_{n}\right)\right)^{2}}{\varphi^{2}\left(x_{n}\right)-\left|x^{\prime \prime}\right|^{2}}} d x^{\prime \prime} d x_{n},
$$

and by the inequality $\left|x^{\prime \prime}\right|<\sigma \varphi\left(x_{n}\right)$,

$$
\varphi\left(x_{n}\right) \sqrt{\frac{1+\left(\varphi^{\prime}\left(x_{n}\right)\right)^{2}}{\varphi^{2}\left(x_{n}\right)-\left|x^{\prime \prime}\right|^{2}}} \sim 1 .
$$

Therefore

$$
\begin{aligned}
\int_{\partial G_{\delta}^{(n-1)}}\left|\alpha\left(x_{n}\right)\right|^{p} \varphi\left(x_{n}\right) d S_{x} & \sim \int_{0}^{\delta} d x_{n} \int_{\left\{\left|x^{\prime \prime}\right|<\sigma \varphi\left(x_{n}\right)\right\}}\left|\alpha\left(x_{n}\right)\right|^{p} \varphi\left(x_{n}\right) d x^{\prime \prime} \\
& \sim \int_{0}^{\delta}\left|\alpha\left(x_{n}\right)\right|^{p} \varphi^{n-1}\left(x_{n}\right) d x_{n} .
\end{aligned}
$$

Combining this estimate and (4.13) we can conclude that integrals

$$
\int_{\partial G_{\delta}}\left|\alpha\left(x_{n}\right)\right|^{p} \varphi\left(x_{n}\right) d S_{x}, \alpha \in \Lambda_{\alpha},
$$

are uniformly small for all $\alpha \in \Lambda_{\alpha}$ if $\delta>0$ is small enough.

To estimate the function $R \in \Lambda_{R}$ that corresponds to $F \in \mathfrak{M}$ we will use Lemma 4.2. Denote $\partial_{0} G=\left\{x \in \partial G:\left|x^{\prime}\right|=\varphi\left(x_{n}\right), 0<x_{n}<1\right\}$. First we will estimate 
the integral

$$
\int_{\partial_{0} G_{\delta}}|R(x)|^{p} \varphi\left(x_{n}\right) d S_{x}
$$

for some $\delta>0$.

The boundary $\partial_{0} G_{\delta}$ can be covered by a finite number of charts $\partial G_{\delta, \sigma}^{(k)}, k=$ $1,2, \ldots, n-1$ (see (4.14)). Let us prove estimates for $R$ for the chart $\partial G_{\delta, \sigma}^{(n-1)}$. We will use the notation $x=:\left(x^{\prime \prime}, x_{n-1}, x_{n}\right) \in \partial_{0} G_{\delta, \sigma}^{(n-1)}$. Then

$$
x_{n-1}=\sqrt{\varphi^{2}\left(x_{n}\right)-\left|x^{\prime \prime}\right|^{2}},|x "|<\sigma \varphi\left(x_{n}\right) .
$$

Choose $s: 0<s<\sqrt{\varphi^{2}\left(x_{n}\right)-|x "|^{2}}$. Then

$$
R(x)=R\left(x^{\prime}, s, x_{n}\right)+\int_{s}^{x_{n-1}} D_{n-1} R\left(x ", \tau, x_{n}\right) d \tau,
$$

where $D_{n-1}=\frac{\partial}{\partial x_{n-1}}$.

Integrating in $s$ we obtain

$$
\begin{aligned}
|R(x)|^{p} \varphi\left(x_{n}\right) \leq & C_{1} \int_{0}^{\sqrt{\varphi^{2}\left(x_{n}\right)-\left|x^{\prime \prime}\right|^{2}}}\left|R\left(x ", s, x_{n}\right)\right|^{p} d s \\
& +C_{2} \varphi\left(x_{n}\right)\left(\int_{0}^{\sqrt{\varphi^{2}\left(x_{n}\right)-\left|x^{\prime \prime}\right|^{2}}}\left|D_{n-1} R\left(x^{\prime \prime}, \tau, x_{n}\right)\right| d \tau\right)^{p} .
\end{aligned}
$$

For the estimate (4.16) we used that $\sqrt{\varphi^{2}\left(x_{n}\right)-\left|x^{\prime \prime}\right|^{2}} \sim \varphi\left(x_{n}\right)$ if $\left|x^{\prime \prime}\right|<\sigma \varphi\left(x_{n}\right)$. Integrating (4.16) on $\partial_{0} G_{\delta, \sigma}^{(n-1)}$, taking into account $d S_{x} \sim d x " d x_{n}$ and using Hölder's inequality for variable $\tau$, we get the following inequality:

$$
\begin{aligned}
& \int_{\partial_{0} G_{\delta, \sigma}^{(n-1)}}|R(x)|^{p} \varphi\left(x_{n}\right) d S_{x} \\
& \leq C_{1} \int_{0}^{\delta} d x_{n} \int_{\left|x^{\prime \prime}\right|<\sigma \varphi\left(x_{n}\right)} d x " \int_{0}^{\sqrt{\varphi^{2}\left(x_{n}\right)-\left|x^{\prime \prime}\right|^{2}}}\left|R\left(x^{\prime \prime}, s, x_{n}\right)\right|^{p} d s \\
& +C_{2} \int_{0}^{\delta} \varphi^{p}\left(x_{n}\right) d x_{n} \int_{\left\{\left|x^{\prime \prime}\right|<\sigma \varphi\left(x_{n}\right)\right\}} d x^{\prime} \int_{0}^{\sqrt{\varphi^{2}\left(x_{n}\right)-\left|x^{\prime \prime}\right|^{2}}}\left|D_{n-1} R\left(x^{\prime \prime}, \tau, x_{n}\right)\right|^{p} d \tau \\
& \leq C_{1} \int_{G_{\delta}}|R(x)|^{p} d x+C_{2} \varphi^{p}(\delta) \int_{G_{\delta}}\left|D_{n-1} R(x)\right|^{p} d x .
\end{aligned}
$$


Combining with (4.12) we obtain

$$
\begin{aligned}
& \int_{\partial_{0} G_{\delta, \sigma}^{(n-1)}}|R(x)|^{p} \varphi\left(x_{n}\right) d S_{x} \\
& \leq C \varphi^{p}(\delta)\left[\int_{G}\left|\frac{R(x)}{\varphi\left(x_{n}\right)}\right|^{p} d x+\int_{G}\left|D_{n-1} R(x)\right|^{p} d x\right] .
\end{aligned}
$$

Using this estimate and Lemma 4.2 we get finally

$$
\|R\|_{p, \varphi, \partial_{0} G_{\delta}} \leq C \varphi(\delta)\|F\|_{W_{p}^{1}(G)} .
$$

This estimate and corresponding estimates for the integrals of $\left|\alpha\left(x_{n}\right)\right|^{p}$ on $\partial_{0} G_{\delta}$ for $\alpha \in \Lambda_{\alpha}$ allow us to conclude that for any $\epsilon_{2}>0$ there exists such a $\delta_{0}>0$ that for all $\delta \in\left(0, \delta_{0}\right)$ and for all $F \in \mathfrak{M}$ the following inequality is correct:

$$
\|F\|_{p, \varphi, \partial_{0} G_{\delta}}<\epsilon_{2} .
$$

Let $\delta$ be an arbitrary number from $\left(0, \delta_{0}\right)$. Denote by $k_{\delta}$ a function of the class $C^{1}([0,1])$ with the following properties: $0 \leq k_{\delta} \leq 1$ on $[0,1], k_{\delta}(t)=1$ when $\delta<t \leq 1, k_{\delta}(t)=0$ when $0 \leq t \leq \delta / 2$. Denote by $\mathfrak{M}(\delta)$ traces of functions $F$ from $\mathfrak{M}$ on $G \backslash \overline{G_{\delta / 2}}$. The set $\mathfrak{M}(\bar{\delta})$ is bounded in $W_{p}^{1}\left(G \backslash \overline{G_{\delta / 2}}\right)$ and therefore 13 is compactly embedded into $L^{p, \varphi}\left(\partial_{0} G \backslash \partial_{0} G_{\delta / 2}\right) \sim L^{p}\left(\partial_{0} G \backslash \partial_{0} G_{\delta / 2}\right)$ (for fixed $\delta)$. Let $\epsilon>0$ be an arbitrary number. Choose a finite $\epsilon / 2$-network for $\Lambda(\delta)=$ $I_{2}(\mathfrak{M}(\delta)) \subset L^{p}\left(\partial_{0} G \backslash \partial_{0} G_{\delta / 2}\right)$ that is a finite set of functions $\tilde{\nu}_{1}, \ldots, \tilde{\nu}_{r}$. Then the set of functions $\nu_{j}=k_{\delta} \tilde{\nu}_{j}, j=1,2, \ldots, r$ represents a finite $\epsilon / 2$-network for the set $k_{\delta} \Lambda(\delta)=\left\{k_{\delta} F: F \in \mathfrak{M}(\delta)\right\}$ into $L^{p, \varphi}\left(\partial_{0} G\right)$. It is possible to suppose that for the same $\delta$ the inequality (4.19) is correct with $\epsilon_{2}=\epsilon / 2$.

Finally we will prove that the set of functions $\nu_{1}, \ldots, \nu_{r}$ represents an $\epsilon$-network of $\Lambda$ into $L^{p, \varphi}\left(\partial_{0} G\right)$. The argument is standard. Let $F \in \mathfrak{M}$. Then $F=\left(1-k_{\delta}\right) F+$ $k_{\delta} F$. Because $k_{\delta} F \in k_{\delta} \Lambda(\delta)$ there exists such a $\nu_{i}$ that $\left\|k_{\delta} F-\nu_{i}\right\|_{p, \varphi, \partial_{0} G}<\epsilon / 2$. Then by (4.19),

$$
\left\|F-\nu_{i}\right\|_{p, \varphi, \partial_{0} G} \leq\left\|\left(1-k_{\delta}\right) F\right\|_{p, \varphi, \partial_{0} G}+\left\|k_{\delta} F-\nu_{i}\right\|_{p, \varphi, \partial_{0} G}<\epsilon / 2+\epsilon / 2=\epsilon .
$$

Therefore $\nu_{1}, \ldots, \nu_{r}$ is the desired $\epsilon$-network for $\Lambda=I_{2}(\mathfrak{M})$ in $L^{p, \varphi}\left(\partial_{0} G\right)$.

\section{REFERENCES}

[1] Sh.Agmon, Lectures on Elliptic Boundary Value Problems. D.Van Nostrand Co. Princeton, Toronto, New York, London, 1965. MR0178246 (31:2504)

[2] O.V.Becov, V.P.Il'in, S.M.Hikolskii, Integral representation of functions and embedding theorems (Russian), Nauka, 1975. MR0430771 (55:3776)

[3] R.Courant, D.Hilbert, Methoden der mathematischen Physik, vol. 2, Springer-Verlag, Berlin, 1937. MR0009069 (5:97b)

[4] D.Gilbarg, N. Trudinger, Elliptic partial differential equations of second order, Springer, Berlin, 2001. MR.1814364 (2001k:35004)

[5] W.Evans, D.Harris, Sobolev embeddings for generalized ridged domains, Proc. Lond. Math. Soc., 54, No. 3, (1987), 141-175. MR872254 (88b:46056)

[6] V.M.Gol'dshtein, Yu.G. Reshetnyak, Quasiconformal Mappings and Sobolev Spaces, Kluwer Academic Publishers. Dordrecht, Boston, London. 1990. MR1136035 (92h:46040)

[7] V.Gol'dshtein and L.Gurov. Applications of change of variables operators for exact embedding theorems, Integral Equat. Oper. Theory, 19, (1994), 1-24. MR.1271237 (95c:46045)

[8] V.Goldshtein, A.G.Ramm, Compactness of the embedding operators for rough domains, Math. Inequal. and Applications, 4, No. 1, (2001) 127-141. MR.1809846 (2001m:46075) 
[9] V.Goldshtein, A.G.Ramm, Embedding operators and boundary-value problems for rough domains, IJAMM, 1, (2005) 51-72.

[10] O.A. Ladyzhenskaya, N.N. Ural'tseva, Linear and quasilinear elliptic equations, Academic Press, New York, 1968. MR0244627 (39:5941)

[11] V.Maz'ya, Sobolev Spaces, Springer Verlag, Berlin, 1985. MR.817985 (87g:46056)

[12] A.G.Ramm, Inverse problems, Springer, New York, 2004.

[13] S.L.Sobolev, Some applications of functional analysis to mathematical physics. Leningrad, Leningrad State Unuversity, 1950.

[14] M.Ju.Vasiltchik, Traces of functions from Sobolev space $W_{p}^{1}$ for domains with non Lipschitz boundaries. In: Modern problems of geometry and analysis. Novosibirsk, Nauka, (1989), 9-45.

[15] M.Ju.Vasiltchik, Necessary and sufficient conditions on traces of functions from Sobolev spaces for a plane domain with non Lipschitz boundaries. In: Studies on mathematical analysis and Riemannian geometry. Novosibirsk, Nauka, (1992), 5-29.

[16] M.Ju.Vasiltchik, V.M.Gol'dshtein, About solvability of third boundary-value problem for domains with peak. (Russian) Matematicheskie zametki, 78, 3, (2005), 466-468. MR.2227517

[17] W.P.Ziemer, Weakly Differentiable Functions, Sobolev spaces and functions of bounded variation, Graduate Texts in Mathematics, vol. 120, Springer-Verlag, New York, 1989. MR.1014685 (91e:46046)

[18] P. Grisvard, Elliptic problems in nonsmooth domains, Pitman, Boston, MA, 1985, 410 pp. MR775683 (86m:35044)

Department of Mathematics, Ben Gurion University of the Negev, P. O. Box 653, Beer Sheva, 84105, IsRael

E-mail address: vladimir@bgu.ac.il

Department of Mathematics, Novosibirsk Technical University, Novosibirsk, Russia 